\title{
Quantum computation on gate-defined semiconductor quantum dots
}

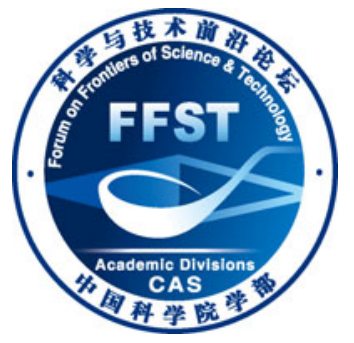

\author{
LI HaiOu, YAO Bing, TU Tao \& GUO GuoPing* \\ Key Laboratory of Quantum Information, Chinese Academy of Sciences, University of Science and Technology of China, Hefei 230026, China
}

Received December 12, 2011; accepted January 16, 2012

During the past few years, researchers have made significant progress on quantum information processing in gate controlled semiconductor quantum dots. We review the global research efforts, including works by our group, which provides pathways towards applications in quantum computation.

quantum dot, quantum computing

Citation: $\quad$ Li H O, Yao B, Tu T, et al. Quantum computation on gate-defined semiconductor quantum dots. Chin Sci Bull, 2012, 57: 1919-1924, doi: $10.1007 / \mathrm{s} 11434-012-5091-5$

The emergence of the computer has changed the human lifestyle greatly, and the development of science is increasing the demand for more powerful computers. The processing power of a classical computer depends on the number of transistors on the microprocessor. Thus, to improve the speed of the computer, one should place as many transistors as possible on each microprocessor. However, this approach is limited by the physical constraints on transistors. Specifically, quantum effects pose difficult obstacles for further decreasing the size of transistors. This suggests the idea that it may be possible to exploit these quantum phenomena for computing purposes, by building a processor whose function relies on coherent quantum behaviors. Here comes the quantum computer.

Quantum computation is the study of information processing tasks that can be accomplished using quantum mechanical systems. There are five basic requirements for realizing quantum computation in physical devices [1]: (1) Qubit. A two-level quantum system is called a quantum bit, or qubit. The two eigenstates $\mid 0>$ and $\mid 1>$ act as the basic units of the qubit, just like the bits 0 and 1 in classical computation. For practicality, the quantum computer should consist of several hundred qubits, so we need a scalable

*Corresponding author (email: gpguo@ustc.edu.cn) physical system. (2) Initialization. It is important that the qubits are initialized to a known value before starting the computation. (3) Manipulation. Quantum computation is based on the transformation of the quantum state. Thus the system should be able to perform any unitary transformation (a universal set of quantum gate operations). One can obtain the desired states or results through a sequence of gate operations on the initial states. It has been proved that the combination of full single qubit control with the two qubit CNOT gate can result in all possible quantum algorithms. (4) Readout. The system should be able to measure the final states after the unitary transformation of the initial states. (5) Coherence. The quantum computing system should have a long decoherence time, i.e., many gate operations and measurements can be performed before decoherence occurs.

There are several systems with the potential to realize quantum computation. Each system has its own advantages and difficulties. Qubits can be encoded in the states of photons, atoms, nuclear or artificial atoms such as superconductor junctions and semiconductor quantum dots. Some systems can be manipulated conveniently or have long quantum coherence times, while others seem more scalable. We focus on quantum computation in gate controlled semiconductor quantum dots, which is regarded as one of the most promising candidates for quantum computation. This 
system is more attractive than the others in that it offers the advantages of large scale integration and compatibility with current computer technology. In what follows, we will give a brief introduction to this kind of quantum dot. We will then demonstrate the impressive achievements in the rapidly developing field of quantum computation based on gate controlled quantum dots. Some outlooks will be presented in the last section.

\section{Gate controlled semiconductor quantum dots}

Quantum dots are also called "artificial atoms" [2], with a typical size ranging from several nanometers to several microns. The movement of electrons is restricted due to size limitations on quantum dots, which results in discrete energy levels. Common quantum dots can be divided into three groups: colloidal quantum dots, self-assembled quantum dots and gate controlled quantum dots [3]. In this paper, we mainly discuss the last of these. A gate controlled quantum dot is a two dimensional electron gas with some electrodes attached, and is based on the relatively mature semiconductor micro-nanofabrication method. A lateral gate defined quantum dot is depicted in Figure 1.

(i) Single quantum dot. The production of quantum dots requires preparing high quality chips from GaAs/AlGaAs heterostructure by Molecule Beam Epitaxy (MBE), manufacturing the top gates by E-beam lithography (EBL) and evaporation (EV), and bringing the source and drain electrodes into contact with the electron gas (Ohmic contact) by annealing. The electrons of the conduction band gather at the interface between GaAs and AlGaAs, and can only move laterally. If we apply a negative voltage to the gate electrodes, the two dimensional electron gas directly under the gate electrode will be pushed away and form a confined region for the electrons, as shown in Figure 1. Gate controlled quantum dots are considered to be one of the most plausible systems for quantum computation since it is easy to control their shape, size and interaction strength precisely [4]. It is also obvious that this kind of quantum dot is scalable.

(ii) Double quantum dot. A double quantum dot is also

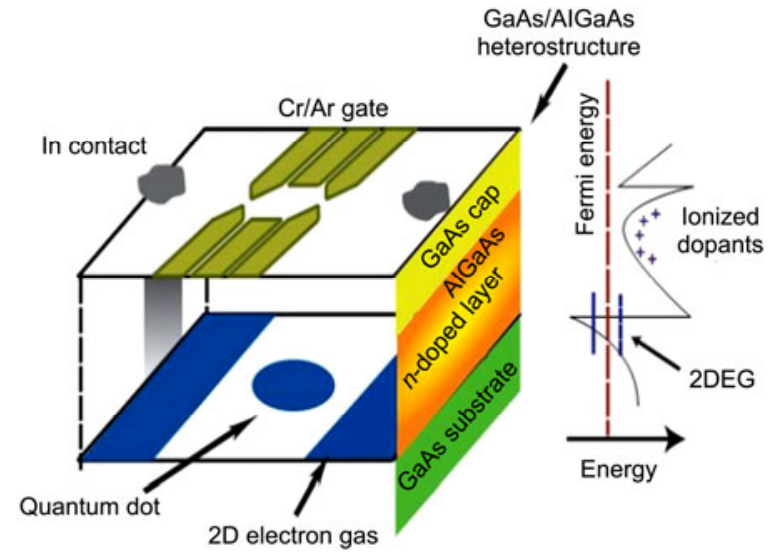

Figure 1 (Color online) Schematic diagram of GaAs/AlGaAs lateral quantum dot.

called an "artificial molecule", and provides a good platform for research on the interaction between artificial atoms [5]. As shown in Figure 2, there are two kinds of double quantum dot which have been prepared in our group. We would like to emphasize that this is the first effort involving gate controlled quantum dots in China [6].

\section{Spin based quantum computation in gate controlled quantum dots}

In 1998, Loss from Bessel University and Divincenzo from IBM raised the idea of spin based quantum computation in semiconductor quantum dots [7]. Because of weak interactions with the environment, the spin state is seldom disturbed, resulting in a relatively long decoherence time even in the solid phase. The semiconductor quantum dot system is convenient for large scale integration, and is compatible with the present technology. Because of these features, quantum computation based on electron spin in the semiconductor quantum dot is the direction with the most potential in the future.

(i) Electron spin qubit. Loss and Divincenzo proposed encoding the qubit based on a single electron spin, i.e. the
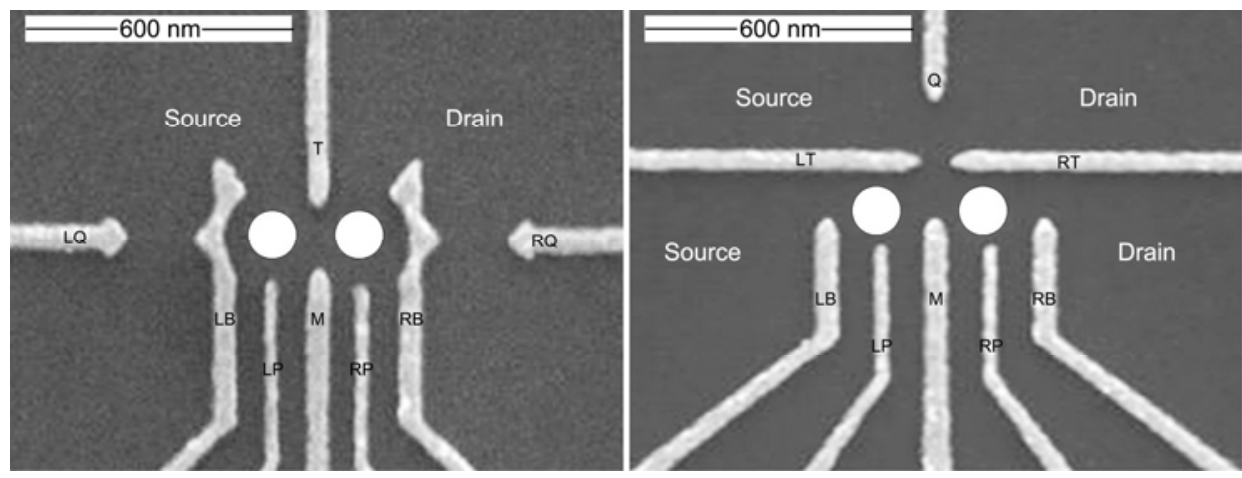

Figure 2 Scanning electron micrograph of GaAs/AlGaAs gate controlled double quantum dot in series (by USTC group). 
qubits $\mid 0>$ and $\mid 1>$ are represented by the states of spin up $\mid \uparrow>$ and spin down $\mid \downarrow>$. When there is only one electron in a quantum dot, the system is similar to a hydrogen atom. When an external magnetic field is applied, all orbits can split into a two-level structure because of the existence of electron spin. The state of spin up is parallel to the direction of the magnetic field and is the ground state, while the excited state is spin down and is anti-parallel to the magnetic field. The energy difference between these states is Zeeman energy.

(ii) Initialization of electron spin qubit. It is now possible to initialize electron spin states. In 2003, Kouwenhoven and Vandersypen's team at Delft University placed a quantum dot in a dilution refrigerator with an environment temperature of $15 \mathrm{mK}$ and applied a strong magnetic field of $10 \mathrm{~T}$ to the system [8]. This allowed the Zeeman energy of the quantum dot to be $200 \mu \mathrm{eV}$, which is much larger than the thermal energy of $25 \mu \mathrm{eV}$ and smaller than interval between the orbital energy of $1.1 \mathrm{meV}$ and the charging energy of $2.5 \mathrm{meV}$. Thus, the electron can have two states of spin up $\mid \uparrow>$ and spin down $\mid \downarrow>$, which can be regarded as the qubits $\mid 0>$ and $\mid 1>$. This is the first time that electron spin qubits have been prepared and characterized experimentally.

(iii) Gate operations of electron spin qubits. In 2006, Kouwenhoven and Vandersypen's team applied a microwave pulse with a frequency that could resonate with the Zeeman energy level of the quantum dot system to realize a single qubit operation [9]. They demonstrated that this oscillating magnetic field is perpendicular to the static one, resulting in rotation of the electron spin in the time domain, i.e. $\mid \uparrow>$ changes to $\mid \downarrow>$. This method is also called the electron spin resonance (ESR) technique and the result is also called Rabi oscillation.

In 2005, the Marcus and Yacoby groups at Harvard University applied electrical pulse techniques to realize the coupling of two electron spins in double quantum dots [10]. The two electron spins in the close quantum dots are coupled by a tunneling barrier, which can be expressed by the Hamiltonian of the Heisenberg exchange interaction. The interaction strength depends on the overlap between the wave functions of the two electrons, and can be controlled by the gate electrodes. Thus the spins of the two electrons can be swapped or even entangled by adjusting the time the pulse is applied to the gate electrodes. For example, they demonstrated the SWAP operation $\mid \uparrow \downarrow>$ to $\mid \downarrow \uparrow>$ for two adjacent spins in $180 \mathrm{ps}$.

By combining the rotation operation of a single electron spin and the interaction operation of two electron spins, one can realize any quantum gate operation of electron spins.

(iv) Measurement of electron spin qubit. One of the critical problems with qubits is how to measure spin states. In 2004, Kouwenhoven and Vandersypen's team tuned the Fermi level of the source and drain between the spin up and spin down states [11]. The electron that is spin down $\mid \downarrow>$ will then tunnel out of the quantum dot while the electron that is spin up $\mid \uparrow>$ will stay in the quantum dot. This difference can be distinguished by the quantum point contact (QPC) electrometer beside the quantum dot.

(v) Long coherence time of electron spin qubit. Decoherence time of the system should be much longer than the operation time of the quantum gate operations. Generally, only if $10^{4}$ operations can be completed during the decoherence time of the system can quantum computation be realized. In the present system, spin relaxation and dephasing both result in the loss of quantum information. The spin relaxation time $T_{1}$ and dephasing time $T_{2}$ are strongly related to the material. Spin relaxation comes mainly from spin-orbit coupling, while spin dephasing comes from the hyperfine interaction between the spin and nuclear spin of order $10^{6}$ around the quantum dots. It has been observed that $T_{1}$ and $T_{2}$ for GaAs/AlGaAs quantum dots are about 1 $\mathrm{ms}$ [11] and $10 \mathrm{~ns}$ [10], respectively. In particular, the dephasing coming from nuclear spin can be reduced by the dynamical decoupling method [12] or dynamical nuclear polarization method [13], and as a result the spin dephasing time $T_{2}$ can be up to $1 \mu \mathrm{s}$, or $10^{5}$ longer than that of the spin SWAP gate operation (about $180 \mathrm{ps).}$

In summary, the basic requirements for quantum computation based on electron spin qubit are fulfilled in both conceptual and practical terms, as illustrated in Figure 3 [14]. These are exciting times for electron spin quantum computing, as it continues to drive a rapidly expanding field that has a promising future [15].

\section{Some outlook of quantum computation in quantum dots}

Based on the significant progress achieved in this research area, we now discuss what may need to be done to investigate the fundamental quantum mechanical processes in solids and provide pathways towards applications in quantum information processing.

(i) New qubits. Many quantum coding methods, such as quantum error correcting codes, decoherence-free subspaces and dynamic decoupling, have been invented to avoid disturbing quantum information. In order to avoid decoherence caused by nuclear spin, in 2005, the Marcus, Yacoby and Lukin groups at Harvard University introduced an alternative coding proposal using spin qubits in two electron singlet and triplet states [16]. In 2009, the Yacoby group demonstrated that a universal single qubit operation could be realized by nuclear spin polarization [17]. Due to the spin blockade effects, the singlet state will transfer while the triplet state remains unchanged and one can realize the measurement of a single qubit with the nearby QPC detector. Furthermore, this coding method protects the qubits from the low frequency noise and reduces the effect of the hyperfine 

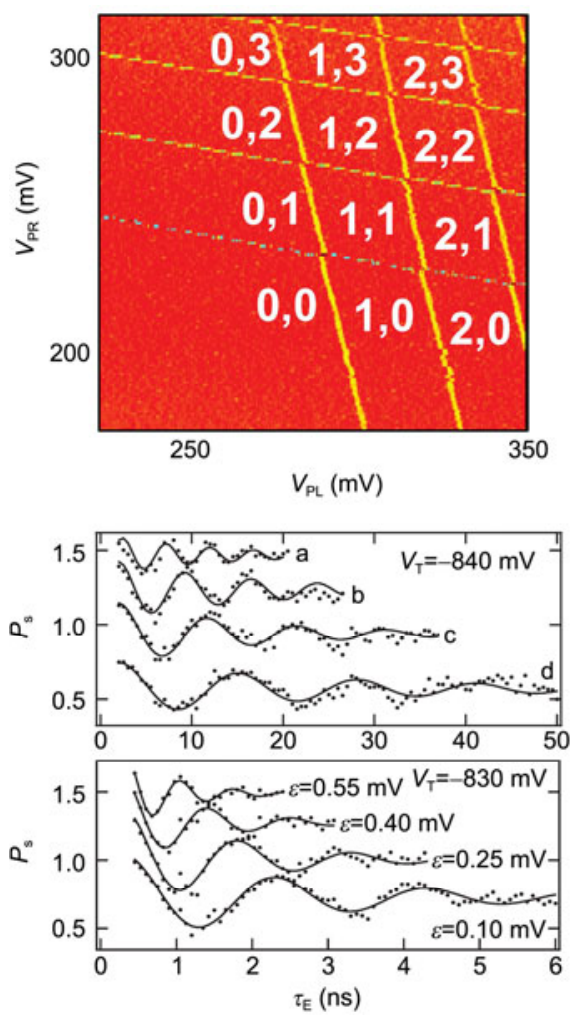
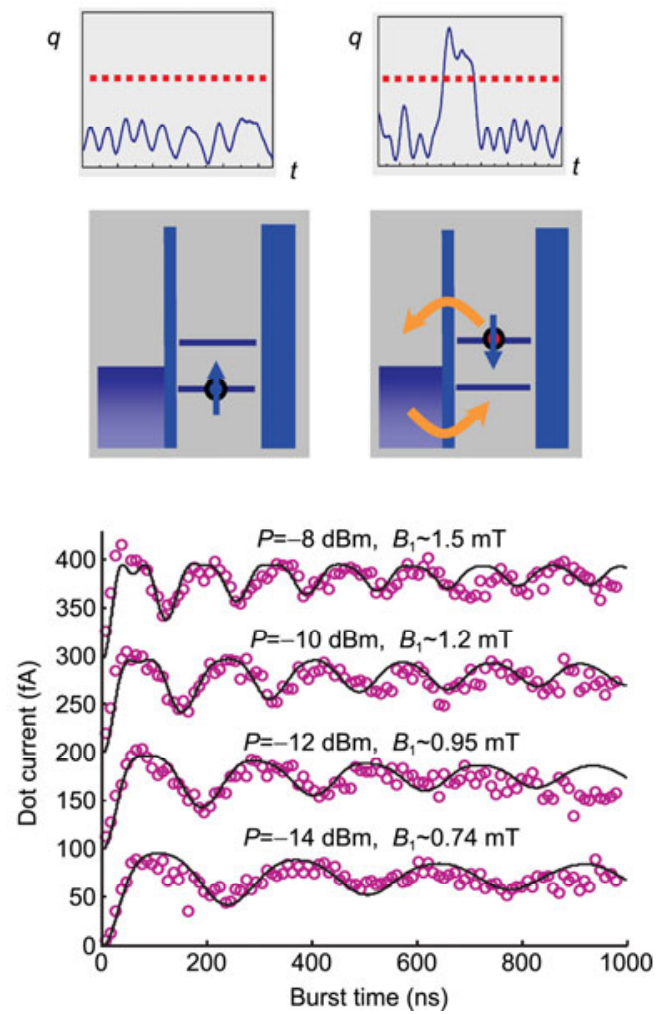

Figure 3 (Color online) Milestones of electron spin qubits in GaAs/AlGaAs quantum dots.

interaction.

(ii) New architectures. Quantum dots can be coupled directly with each other by capacitive interaction. Recently [18], the Marcus group has tried to realize inter-control between the double quantum dots by capacitive coupling as shown in Figure 4. Our group has also proposed achieving quantum information processing in a scalable architecture consisting of a switchable Ising-like interaction between any two adjacent double quantum dot qubits $[19,20]$.

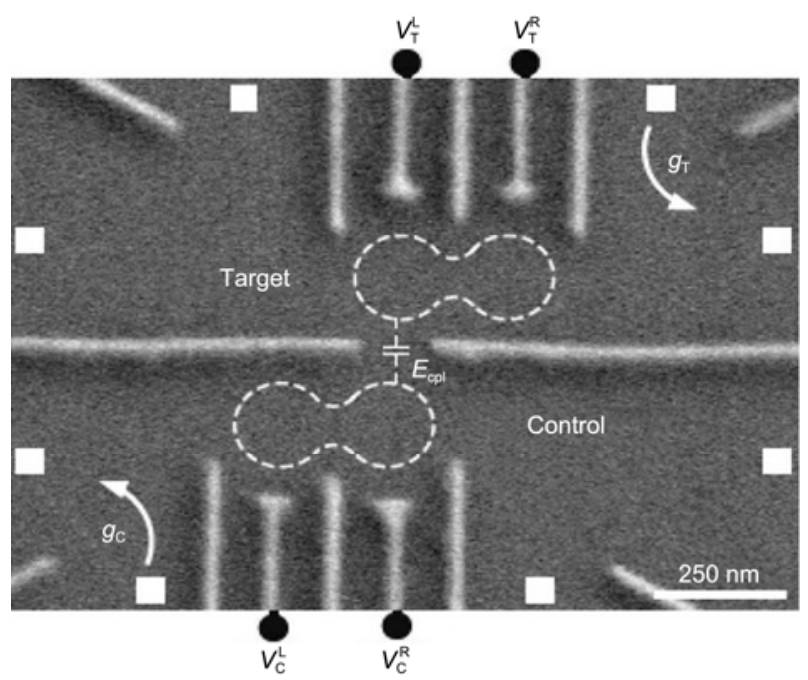

Figure 4 A pair of double quantum dots device.
However, this coupling approach is not easily scalable beyond three or four qubits because of the limitations of the architecture design and the measurement crosstalk. Furthermore, the coupling strength between distant qubits is very weak, while performing gate operations between an arbitrary pair of distant qubits is highly desirable in a quantum computer. An efficient way to achieve this goal is to couple the qubits to a quantum databus, which distributes quantum information among the qubits. As shown in Figure 5 , we proposed placing the double quantum dots on the side of a superconducting transmission line resonator (TLR) [21,22]. The TLR functions as a useful quantum databus, and in this way, we can couple many double quantum dot spin qubits and be able to prepare the desired entangled states. Our proposal is an attractive architecture for quantum information processing on a chip.

(iii) New materials. Most experiments have focused on quantum dots made from III-V semiconductors, such as GaAs/AlGaAs. However, the coherence of electron spins in these materials is limited by hyperfine interactions with nuclear spins. Recently, research efforts have produced quantum dots using new materials such as $\mathrm{Si} / \mathrm{SiO}_{2}$ [23], $\mathrm{Si} /$ $\mathrm{SiGe}$ [24], Ge/Si core/shell nanowires [25], carbon nanotubes, and graphene [26,27], which seem ideally suited to overcome the above limitation as the most abundant nuclei in these new materials have spin zero. For example, Jiang's team at UCLA were the first to find the spin relaxation time $T_{1}$ in $\mathrm{Si} / \mathrm{SiO}_{2}$ quantum dots, with $T_{1}$ equal to approximately 

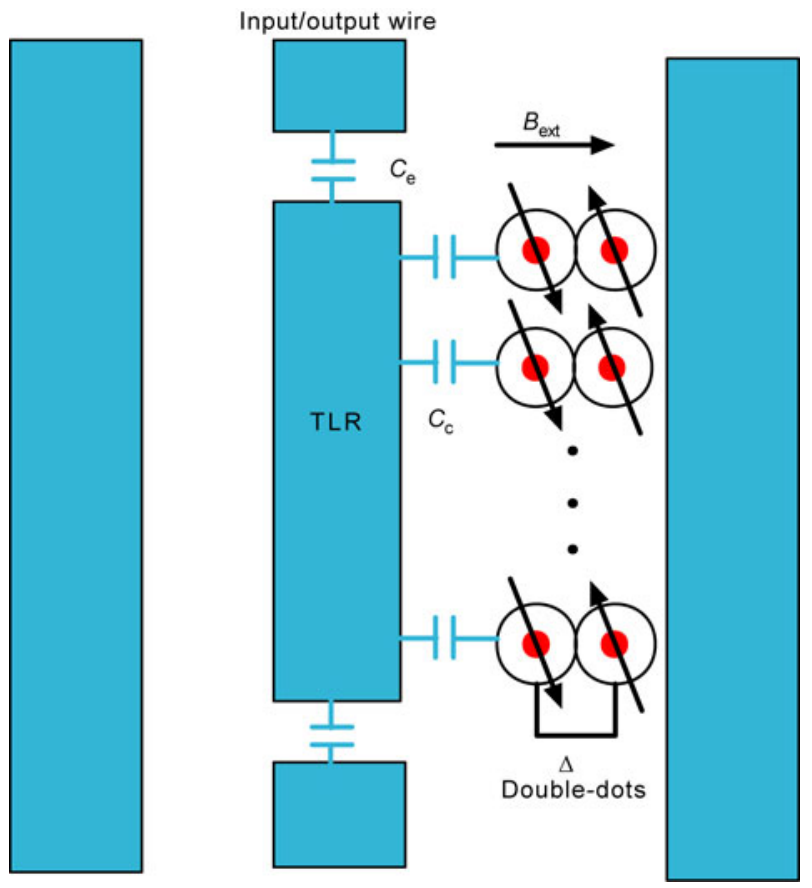

Figure 5 (Color online) Schematic diagram of a TLR and several double quantum dots coupled in circuit (by USTC group).

$1 \mathrm{~ms}[23]$.

Our group has also made progress towards quantum computation on graphene based quantum dot devices. From the theoretical aspect, we proposed forming a one-dimensional graphene quantum dot chain from graphene nanoribbon, so that universal quantum computation can be implemented through always-on interaction between the dots [28]. On the experimental side, we have designed and fabricated the first twin-dot structure in which the larger dot serves as a single electron transistor (SET) to read out the charge state of the nearby gate controlled small dot [29], as shown in Figure 6. Owing to the high SET sensitivity, we can probe Coulomb charging as well as the excited states spectra of graphene quantum dots, even though the current through the graphene quantum dot is too small to be measured by conventional transport means. In addition to graphene double quantum

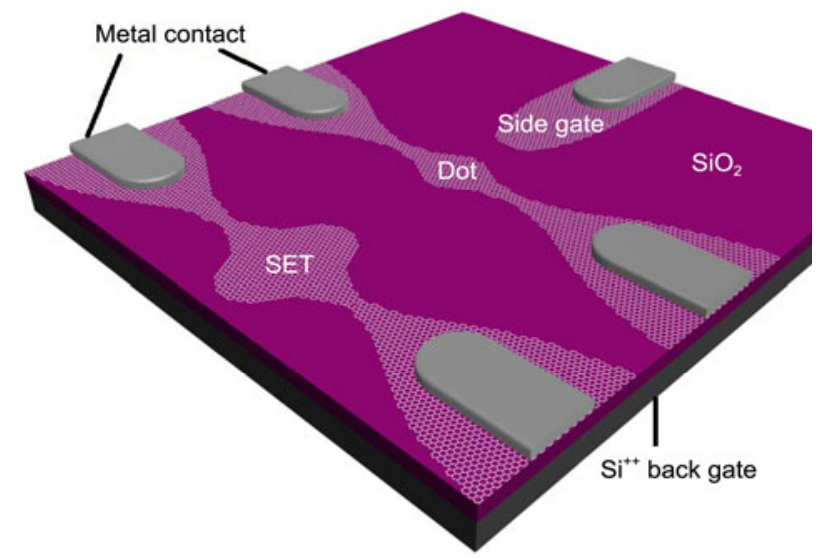

Figure 6 (Color online) Schematic of a representative device with a graphene quantum dot and a nearby SET as a charge sensor (by USTC group).
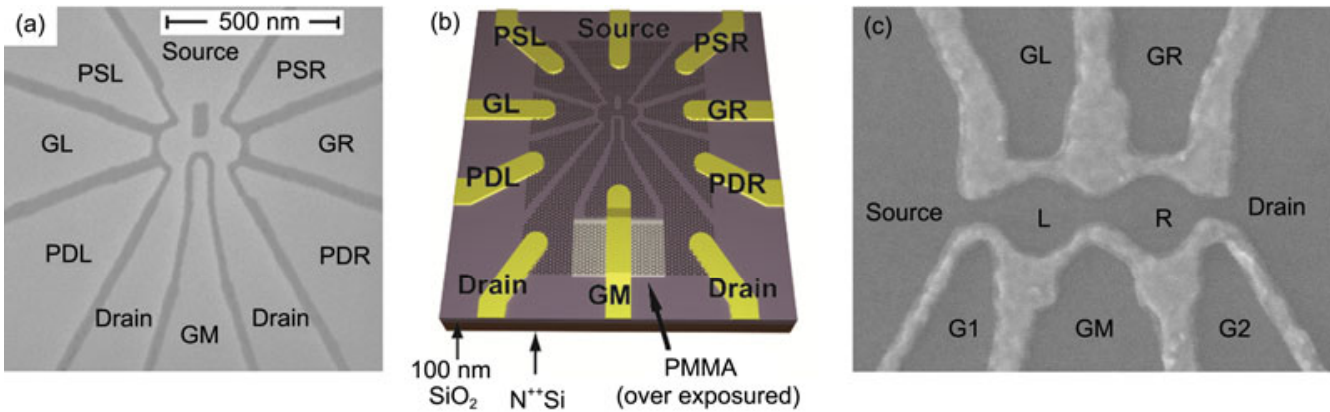

Figure 7 (Color online) Scanning electron micrograph of graphene double quantum dot (a) and in parallel (b), in series (c) (by USTC group).
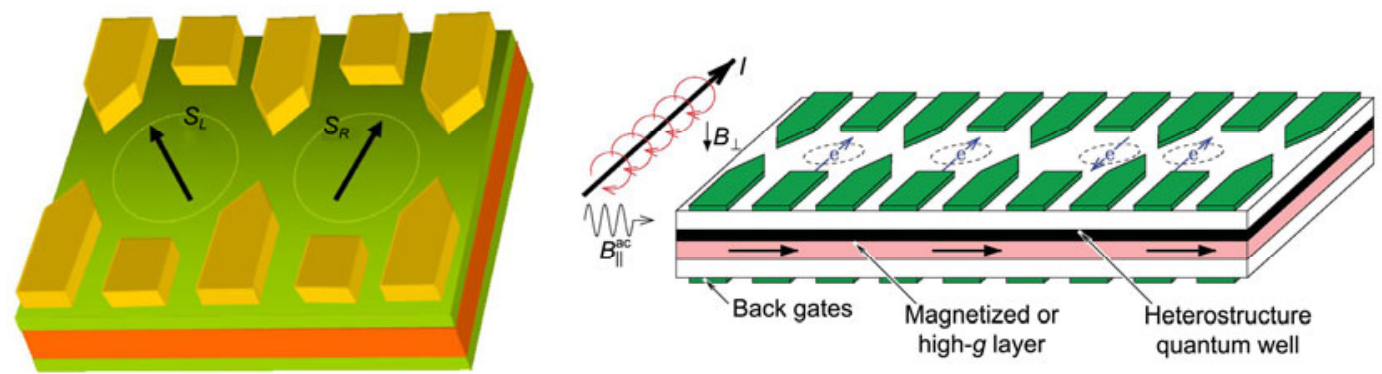

Figure 8 (Color online) There is a long way to go to realize large scale quantum computation from two electron spin qubits. 
dots in series, we reported the first realization of gate controlled parallel coupled graphene double quantum dot in both single layer and bilayer graphene [30], shown in Figure 7. The graphene double quantum dot and integrated charge sensor serve as an essential building block for forming a solid-state qubit free of nuclear spin.

In conclusion, what was considered almost impossible a decade ago, in both conceptual and practical terms, is now a reality. Electron spin qubits can be isolated, initialized, coherently manipulated and read out using electrical techniques. All the requirements for quantum computation based on electron spins in gate controlled quantum dots can be realized in principle, but there is still a long way to go. Much more work is needed before a real quantum computer is produced [31], shown in Figure 8.

This work was supported by the National Basic Research Program of China (2011CBA00200) and the National Natural Science Foundation of China (10934006, 11074243 and 91121014$)$.

1 Nielsen M A, Chuang I L. Quantum Computation and Quantum Information. New York: Cambridge University Press, 2000

2 Kouwenhoven L, Marcus C. Quantum dots. Phys World, 1998, 6: $35-40$

3 Kastner M A. The single-electron transistor. Rev Mod Phys, 1992, 64: $849-858$

4 DiVincenzo D P. Double quantum dot as a quantum bit. Science, 2005, 309: 2173-2174

5 Van der Wiel W G, DeFranceschi S, Elzerman J M, et al. Electron transport through double quantum dots. Rev Mod Phys, 2003, 75: $1-22$

$6 \mathrm{Cao}$ G, $\mathrm{Li} \mathrm{H} \mathrm{O}$, Tu T, et al. Electron states in parallel double quantum dots with a tunable inter-dot coupling. Chin Phys Lett, 2009, 26: 097302

7 Loss D, DiVincenzo D P. Quantum computation with quantum dots. Phys Rev A, 1998, 57: 120-126

8 Hanson R, Witkamp B, Vandersypen L M K. Zeeman energy and spin relaxation in a one-electron quantum dot. Phys Rev Lett, 2003, 91: 196802

9 Koppens F H L, Buizert C, Tielrooij K J, et al. Driven coherent oscillations of a single electron spin in a quantum dot. Nature, 2006, 442: 766-771

10 Petta J R, Johnson A C, Taylor J M, et al. Coherent manipulation of coupled electron spins in semiconductor quantum dots. Science, 2005, 309: 2180-2184

11 Elzerman J M, Hanson R, Willems van Beveren L H, et al. Single- shot read-out of an individual electron spin in a quantum dot. Nature, 2004, 430: 431-435

12 Bluhm H, Folleti S, Neder I, et al. Dephasing time of GaAs electron-spin qubits coupled to a nuclear bath exceeding $200 \mu \mathrm{s}$. Nat Phys, 2011, 7: 109-113

13 Reilly D J, Taylor J M, Petta J R, et al. Suppressing spin qubit dephasing by nuclear state preparation. Science, 2008, 321: 817-820

14 Hanson R, Kouwenhoven L P, Petta J R, et al. Spins in few-electron quantum dots. Rev Mod Phys, 2007, 79: 1217-1265

15 Hanson R, Awschalom D D. Coherent manipulation of single spins in semiconductors. Nature, 2008, 453: 1043-1049

16 Taylor J M, Engel H A, Dur W, et al. Fault-tolerant architecture for quantum computation using electrically controlled smiconductor spins. Nat Phys, 2005, 1: 177-183

17 Folleti S, Bluhm H, Mahalu D, et al. Universal quantum control of two-electron spin quantum bits using dynamic nuclear polarization. Nat Phys, 2009, 5: 903-908

18 Van Weperen I, Armstrong B D, Laird E A, et al. Charge-states conditional operation of a spin qubit. Phys Rev Lett, 2011, 107: 030506

19 Guo G P, Zhang H, Tu T, et al. One-step preparation of cluster states in quantum-dot molecules. Phys Rev A, 2007, 75: 050301

20 Zhang H, Guo G P, Tu T, et al. Quantum computation and Bell-state measurements with double dot molecules. Phys Rev A, 2007, 76: 012335

21 Lin Z R, Zhu F Y, Tu T, et al. Generation of quantum-dot cluster states with superconducting transmission line resonator. Phys Rev Lett, 2008, 101: 230501

22 Guo G P, Zhang H, Hu Y, et al. Dispersive coupling between the superconducting transmission line resonator and the double quantum dots. Phys Rev A, 2008, 78: 020302

23 Xiao M, House M G, Jiang H W. Measurement of the spin relaxation time of single electron in a silicon metal oxide semiconductor based quantum dot. Phys Rev Lett, 2010, 104: 096801

24 Shaji N, Simmons C B, Thalakulam M, et al. Spin blockade and lifetime-enhanced transport in a few-electron $\mathrm{Si} / \mathrm{SiGe}$ double quantum dot. Nat Phys, 2008, 4: 540-544

$25 \mathrm{Hu}$ Y J, Churchill O H, Reilly D J, et al. A Ge/Si heterostructure nanowire-based double quantum dot with integrated charge sensor. Nat Nanotech, 2007, 2: 622-625

26 Ponomarenko L A, Schedin F, Katsnelson M I, et al. Chaotic Dirac billiard in graphene quantum dots. Science, 2008, 320: 356-358

27 Wang L J, Cao G, Tu T, et al. Ground states and excited states in a tunable graphene quantum dot. Chin Phys Lett, 2011, 28: 067301

28 Guo G P, Lin Z R, Li X P, et al. Quantum computation with graphene nanoribbon. New J Phys, 2009, 11: 123005

29 Wang L J, Cao G, Tu T, et al. A graphene quantum dot with a single electron transistor as an integrated charge sensor. Appl Phys Lett, 2010, 97: 262113

30 Wang L J, Guo G P, Wei D, et al. Gates controlled parallel-coupled double quantum dot on both single layer and bilayer graphene. Appl Phys Lett, 2011, 99: 112117

31 Ladd T D, Jelezko F, Laflamme R, et al. Quantum computers. Nature, 2010, 464: 45-53

Open Access This article is distributed under the terms of the Creative Commons Attribution License which permits any use, distribution, and reproduction in any medium, provided the original author(s) and source are credited. 